\title{
Tailoring multi-loop atom interferometers with adjustable momentum transfer
}

\author{
L. A. Sidorenkov, ${ }^{*}$ R. Gautier, M. Altorio, R. Geiger, ${ }^{\dagger}$ and A. Landragin \\ LNE-SYRTE, Observatoire de Paris-Université PSL, CNRS, \\ Sorbonne Université, 61 avenue de l'Observatoire, 75014 Paris, France.
}

(Dated: November 24, 2020)

\begin{abstract}
Multi-loop matter-wave interferometers are essential in quantum sensing to measure the derivatives of physical quantities in time or space. Because multi-loop interferometers require multiple reflections, imperfections of the matter-wave mirrors create spurious paths that scramble the signal of interest. Here we demonstrate a method of adjustable momentum transfer that prevents the recombination of the spurious paths in a double-loop atom interferometer aimed at measuring rotation rates. We experimentally study the recombination condition of the spurious matter waves, which is quantitatively supported by a model accounting for the coherence properties of the atomic source. We finally demonstrate the effectiveness of the method in building a cold-atom gyroscope with a single-shot acceleration sensitivity suppressed by a factor of at least 50. Our study will impact the design of multi-loop atom interferometers that measure a single inertial quantity.
\end{abstract}

Matter-wave interference is a central concept of quantum mechanics with a myriad of applications making use of electrons [1], neutrons [2], or atoms and molecules [3]. Examples of applications range from bacteria characterization [4] and bio-molecular analysis [5], to fundamental physics tests [6] and accurate inertial sensing [7]. In most cases, the signal of interest can be detected as it shifts the phase of a sinusoidal interference fringe pattern of two partial waves. However, the presence of auxiliary interferometic loops due to the imperfection of the mirrors results in a multiple-wave interference, which reduces the interference contrast and the phase measurement accuracy.

Light-pulse atom interferometers employ a train of laser pulses that split, deflect and recombine the atomic waves to enclose a single loop Mach-Zehnder interferometer, in the simplest case. Here the light pulses act as atom optical beam splitters and mirrors, respectively. Oftentimes, one may be interested in field derivatives rather than the fields themselves (e.g. gradients of the gravitational field or curvature of a magnetic field), or in a selective measurement in a given frequency band. This is realized with interferometers consisting of several loops $[8,9]$, realized by multiple deflection of the matter-waves with additional mirrors - a technique analogous to the multi-pulse magnetic resonance spectroscopy [10].

The atom-optics relies on coherent atom-light interaction, whose efficiency is limited by the homogeneity of the effective Rabi coupling that depends on the local laser intensity and velocity of the atom. The challenge arises when the non-zero transmission of the atomic mirrors leads to leakage of the matter-waves, which are redirected by subsequent mirrors and eventually form undesired additional interferometer loops, thus degrading the two-wave nature of the interferometer [11]. Understanding and controlling the recombination of these spurious paths is intimately linked to the coherence of the

\footnotetext{
* leonid.sidorenkov@gmail.com

† remi.geiger@obspm.fr
}

matter-wave source, and requires a tailored design of the interferometric sequence and atomic mirrors.

In this Letter, we report on a method which prevents the recombination of spurious paths in multi-loop coldatom interferometers using mirrors that transfer an adjustable momentum to the atom. The high degree of control of this method, compared to other techniques in matter-wave interferometry, enables a detailed study of the recombination of wave-packets. We show that the method of adjustable momentum transfer (AMT) allows for building a pure-rate gyroscope (i.e. fully sensitive to rotation rate and insensitive to acceleration), as proposed in Ref. [12]. Our result can be generalized to atominterferometer sensors of arbitrary multi-loop architectures.

We implement the AMT method in a double-loop atom interferometer aimed at measuring rotation rates and described in Refs. [13, 14]. In short, we laser-cool Cesium atoms in a single internal state $|F=4\rangle$ to the temperature of $1.8 \mu \mathrm{K}$, and launch them vertically using moving molasses in an atomic fountain. The atom optics employ stimulated Raman transitions at $852 \mathrm{~nm}$ that couple the $|F=3\rangle$ and $|F=4\rangle$ internal states with two counter-propagating laser fields of wave-vectors $\vec{k}_{3}$ and $\vec{k}_{4}$, imparting a momentum $\hbar \vec{k}_{\text {eff }}=\hbar\left(\vec{k}_{3}-\vec{k}_{4}\right)$ to the diffracted part of the wave-packet [15]. The interferometric sequence comprising four Raman laser pulses of $\pi / 2, \pi, \pi, \pi / 2$ Rabi angles, forms a symmetric doubleloop interferometer. Since the momentum of the atoms is entangled with their internal state, the accumulated atomic phase difference is read out from the final population difference of the two hyperfine states, as determined by fluorescence detection [16].

Two pairs of retro-reflected Raman beams interact with the atomic cloud at different height as shown in Fig. 1(a). The two mirrors are parallel to each other to better than $0.4 \mu \mathrm{rad}$ (see Ref. [17] for the alignment procedure). The normal to the mirrors, which sets the direction of the effective wave-vector $\vec{k}_{\text {eff }}$, is inclined by an angle $\theta_{0}=3.8^{\circ}$ with respect to the horizontal direction $\hat{x}$ (perpendicular to gravity), in order to lift the 
(a)

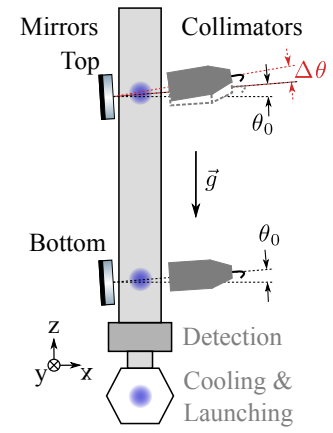

(b)

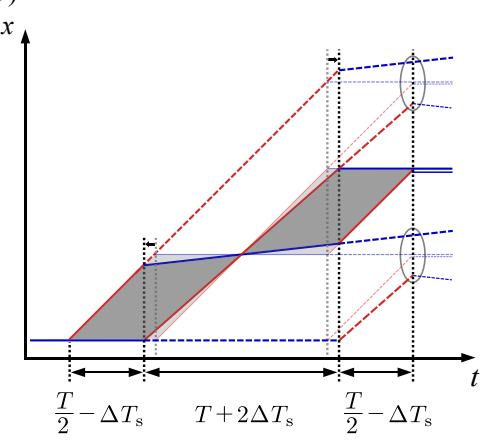

Figure 1. (a) Schematics of the cold-atom gyroscope sensor. The angular tilt $\Delta \theta$ of the top collimator allows for adjusting the effective momentum transfer of the $\pi$-pulses. (b) Space-time diagram (not to scale) of the time-symmetric fourpulse interferometric sequence in the original equal- $k_{\text {eff }}$ (thin half-transparent lines) and AMT (thick full lines) cases. Red (blue) color labels $F=3(F=4)$ internal state of the atoms. Solid (dashed) trajectories correspond to the main (spurious) interferometers. For equal- $k_{\text {eff }}$ (AMT) sequence: the vertical dashed gray (black) lines indicate the timings of the pulses; light- (dark-) gray areas highlight the two loops of the main interferometer. Gray ovals mark the spatial separation of the spurious wave-packets at the last pulse in the AMT sequence. For clarity, we show only the output ports labeled by the $F=4$ state.

degeneracy between the $\pm \hbar k_{\text {eff }}$ transitions owing to the Doppler effect. The top collimator can be further inclined by a small adjustable angle $\Delta \theta \lesssim 20 \mathrm{mrad}$, leading to a reduced modulus of the effective Raman wave-vector of the top beam, $k_{\text {eff }}^{(\mathrm{T})}$, with respect to the bottom one, $k_{\text {eff }}^{(\mathrm{B})} \equiv k_{\text {eff }}$, without changing its direction:

$$
k_{\mathrm{eff}}^{(\mathrm{B})}-k_{\mathrm{eff}}^{(\mathrm{T})}=\epsilon k_{\mathrm{eff}} \approx \frac{\Delta \theta^{2}}{2} k_{\mathrm{eff}} .
$$

This scheme, where the two wave-vectors are not equal (as theoretically studied in Ref. [18] in the context of recoil frequency measurements) allows us to reach the necessary change in the momentum transfer to prevent the recombination of spurious paths in multi-loop interferometers with cold-atom sources. Adjusting the momentum transfer could also be realized by shifting the frequencies of the lasers, as proposed in Ref. [19] and implemented in Refs. [20-22] to reduce systematic errors in single-loop gravity sensors. However, this would require frequency changes of tens of $\mathrm{GHz}$, which makes it impractical, here.

In the traditional double-loop sequence $[11,13]$ (Fig. 1(b), thin half-transparent lines), the four Raman laser pulses are separated by time intervals $T / 2, T$ and $T / 2$, with $T=400 \mathrm{~ms}$. The time symmetry of this sequence with respect to the apogee of the atomic trajectory (crossing of the two loops at $t=t_{1}+T, t_{1}$ being the timing of the first pulse with respect to the launch) leads to a vanishing sensitivity to constant linear acceleration, which is required to build a pure-rate gyroscope. However, two spurious Ramsey-Bordé-like [23] interfer-

ometers (thin dashed lines in Fig. 1(b)) recombine simultaneously with the main one and, having different inertial sensitivity, impair the signal of interest. Distinguishing the spurious interferometers from the main one would require a position-sensitive detector (along the $\hat{x}$ direction) and an atomic source with sub-recoil temperature, which would add complexity to the sensor architecture.

To circumvent this problem, one may apply a small asymmetric time shift of the mirror pulses (both pulses delayed or advanced by $\Delta T_{\mathrm{a}}$ ) [11], inducing sufficient spatial separation of the spurious wave-packets while barely modifying the rotation-rate sensitivity of the main interferometer. Braking its time symmetry, nonetheless, imbalances the space-time areas of the two loops, which causes a sensitivity to constant linear acceleration.

The thick solid trajectory lines in Figure 1(b) show the interferometer sequence using AMT as explored in this Letter. In order to close the main interferometer, the reduction of the momentum transfer at the $\pi$-pulses governed by Eq. (1) is compensated with their shift in time (see Fig. 1(b)) of

$$
\Delta T_{s}=\frac{T \epsilon}{2(1-\epsilon)}
$$

The new degree of freedom provided by AMT allows us to retain the original time symmetry of the main interferometer and to prevent the recombination of the spurious wave-packets, on which we will focus in the following.

In order to sufficiently separate the recombination time of the spurious interferometers from that of the main one (see Supplemental Material, section S3 [24]), we shift the mirror pulses by a fixed time interval $\Delta T=40 \mu \mathrm{s}$ as shown in Figure 2(a). We also deliberately enhance the amplitudes in the spurious branches by changing the Rabi angles of these pulses from $\pi$ to $\pi / 2$, thus making the mirrors half-transparent. Finally, we introduce a controlled variable delay of the third pulse, $\Delta T_{3}$ (see Fig. 2(a)), that allows us to probe the efficiency of the recombination of the spurious interferometers.

In Figure 2(b) we present the evolution of the contrast of the spurious interferometers as we gradually transform the sequence with increasing value of the angle $\Delta \theta$. For each angle, we probe the spurious signal by scanning the value of $\Delta T_{3}$ (blue dots). At $\Delta \theta=0$, we find the maximum of the contrast, as expected, around $\Delta T_{3}=0 \mu \mathrm{s}$. We observe a reduction of the maximum contrast while increasing $\Delta \theta$ towards an almost full suppression around $\Delta \theta=12 \mathrm{mrad}$, followed by a clear revival and a final decay at large angles. To connect the observed contrast behavior with the coherence properties of the cold-atom source, we derive the phase shifts of the bottom (B) and top (T) spurious interferometers (see Supplemental Material, section S1 [24]) as:

$$
\begin{aligned}
& \Delta \Phi^{(\mathrm{B})}=\Delta \Phi_{r}\left(r_{0}\right)+\Delta \Phi_{v}\left(v_{0}\right)+\Delta \Phi^{\prime}-\omega_{R} T \epsilon \\
& \Delta \Phi^{(\mathrm{T})}=\Delta \Phi_{r}\left(r_{0}\right)+\Delta \Phi_{v}\left(v_{0}\right)+\Delta \Phi^{\prime}+\omega_{R} T \epsilon,
\end{aligned}
$$


(a)

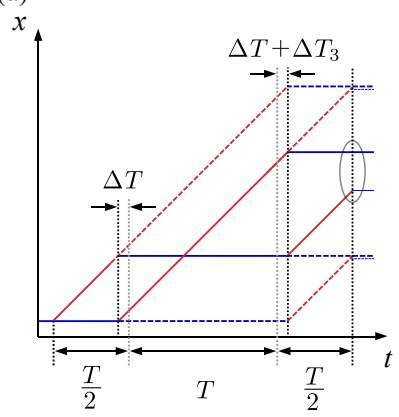

(b)

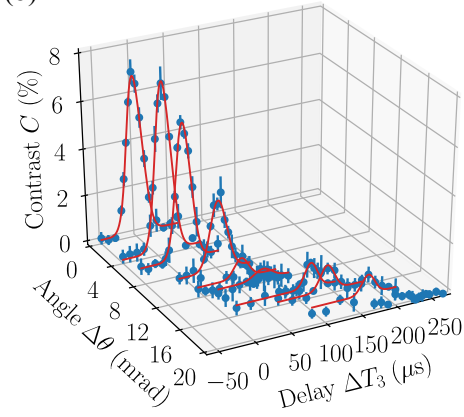

(c)

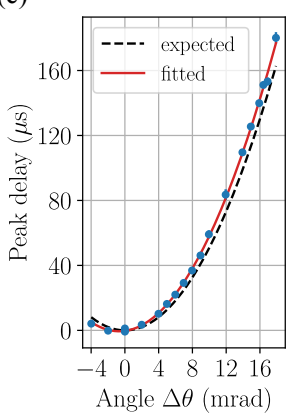

(d)

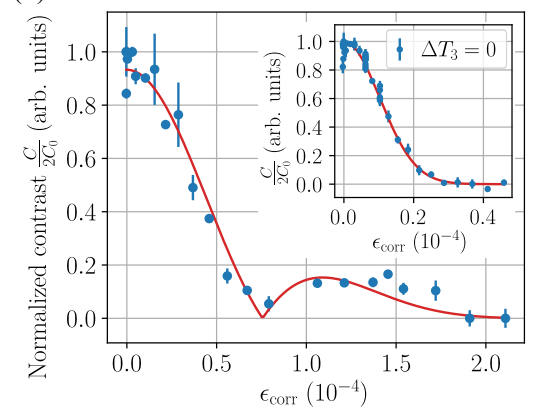

Figure 2. (a) Space-time diagram of the interferometer to introduce the definitions of the fixed $\Delta T=40 \mu \mathrm{s}$ and the variable $\Delta T_{3}$. (b) Peak-peak contrast of the spurious interferometers (blue dots) as a function of the third pulse delay $\Delta T_{3}$, for a set of angles $\Delta \theta$, and Gaussian fits (solid red lines) to the corresponding data. (c) Fitted values of $\Delta T_{3}$ yielding maximum contrast for the probed values of $\Delta \theta$. Dashed black line is the expectation of $\Delta T_{3}=2 \epsilon\left(T+t_{1}\right)$, solid red line is the fit to the data accounting for initial angular offsets $\Delta \theta_{0 z}, \Delta \theta_{0 y}$. (d) Normalized fitted peak contrast for the probed values of $\epsilon_{\mathrm{corr}}=\frac{1}{2}\left(\left(\Delta \theta-\Delta \theta_{0 z}\right)^{2}-\Delta \theta_{0 y}^{2}\right)$. The solid red line is the fit with Eq. 5 with $\sigma_{r}$ and $C_{0}$ as free parameters (see text). Inset: contrast decay for $\Delta T_{3}=0$. The solid red line is the expectation for the measured value of $\sigma_{v}$ and the fitted value of $\sigma_{r}$.

where $\omega_{R} \equiv \frac{\hbar k_{\text {eff }}^{2}}{2 m}$ is the two-photon recoil frequency and

$$
\begin{aligned}
& \Delta \Phi_{r}\left(r_{0}\right)=2 \vec{k}_{\text {eff }} \cdot \vec{r}_{0} \epsilon \\
& \Delta \Phi_{v}\left(v_{0}\right)=\vec{k}_{\text {eff }} \cdot \vec{v}_{0}\left(2 \epsilon\left(T+t_{1}\right)-\Delta T_{3}\right) .
\end{aligned}
$$

We express the phase shifts as a sum of four distinct terms. The first two terms, $\Delta \Phi_{r}\left(r_{0}\right)$ and $\Delta \Phi_{v}\left(v_{0}\right)$, depend on the initial (at launch) position $\vec{r}_{0}=\vec{r}(t=0)$ and velocity $\vec{v}_{0}=\vec{v}(t=0)$ of a given atom in the reference frame of the center of mass of the atomic cloud. The third term, $\Delta \Phi^{\prime}$, incorporates the inertial contribution due to acceleration and the common recoil phase shift. The last term constitutes a relative dephasing of the two spurious interferometers, which increases with $\epsilon$.

The contrast of the spurious interferometric signal, $C\left(\epsilon, \Delta T_{3}\right)$, is given by the (incoherent) sum of the intensities from both interferometers, averaged over the initial statistical velocity and position distributions of the atomic source. We assume uncorrelated Gaussian velocity and position distributions, respectively characterized by the standard deviations $\sigma_{v}$ and $\sigma_{r}$, and obtain (see Supplemental Material, section S2 [24]):

$$
\frac{C\left(\epsilon, \Delta T_{3}\right)}{2 C_{0}}=\left|\cos \left(\omega_{R} T \epsilon\right)\right| e^{-\frac{1}{2}\left(\Delta \Phi_{r}\left(\sigma_{r}\right)^{2}+\Delta \Phi_{v}\left(\sigma_{v}\right)^{2}\right)},
$$

where $C_{0}$ is the maximum mean contrast. The oscillating term reflects the recoil-originated dephasing between the two spurious interferometers, while the exponential suppression factor highlights the role of the finite spatial and momentum spread in the cold-atom source.

The effect of the finite velocity spread on the contrast can be fully eliminated by the proper choice of $\Delta T_{3}=$ $2 \epsilon\left(T+t_{1}\right)$ (from Eq. (4)), which defines the recombination in momentum space and therefore yields the maximum of contrast. This expectation for $\Delta T_{3}$, shown by the dashed black line in Figure 2(c), qualitatively matches the data (blue dots). A quantitative agreement is obtained by accounting for the initial angular mismatch between the collimators in both $z$ (vertical) and $y$ (horizontal) directions via $\epsilon_{\mathrm{corr}}=\left(\left(\Delta \theta-\Delta \theta_{0 z}\right)^{2}-\Delta \theta_{0 y}^{2}\right) / 2[25]$. Fitting the data with $\Delta T_{3}=2 \epsilon_{\mathrm{corr}}\left(T+t_{1}\right)$ (solid red line in Fig. 2(b)) reveals small angular offsets $\Delta \theta_{0 z}=-0.68(5) \mathrm{mrad}$ and $\Delta \theta_{0 y}=1.04(19) \mathrm{mrad}$, which are compatible with the inaccuracy of the initial manual tuning of the collimator of about $1 \mathrm{mrad}$.

In Figure 2(d) we plot the values of fitted maximum contrast of the spurious interferometers normalized to the maximum value among all the datasets, for different values of $\epsilon_{\text {corr }}$. The overall trend, including the zero and the revival, is well reproduced by the fit (solid red line) with the model of Eq. (5) accounting for $\Delta \Phi_{v}=0$ (recombination in momentum space), with $C_{0}$ and $\sigma_{r}$ as free parameters. The fitted value of $\sigma_{r}=0.51(2) \mathrm{mm}$ sets the realistic scale for the spatial extent of the atomic cloud. A non-Gaussian actual cloud shape might be the cause of the slight mismatch around $\epsilon_{\mathrm{corr}} \simeq 1.6 \times 10^{-4}$.

The inset of Figure 2(d) shows the contrast decay in the case of $\Delta T_{3}=0\left(\right.$ where $\left.\Delta \Phi_{v} \neq 0\right)$, which is driven by the finite velocity spread of the source and happens on a much faster $\epsilon$-scale. This behavior does not depend on the specific value of $\Delta T=40 \mu \mathrm{s}$, and is thus also applicable to the case of $\Delta T=\Delta T_{\mathrm{s}}$ (Fig. 1(b)). The complete suppression of the signal of the spurious loops in the time-symmetric AMT sequence therefore happens on a scale of $\epsilon \simeq 4 \times 10^{-5}$. This data is well matched by the expected behavior of Eq. (5) (solid red line), with the value of $\sigma_{v}$ extracted from the widths of the peaks in the panel (b) (see Supplemental Material, section S3 [24]).

We now focus on the main (double-loop) interferometer in the time-symmetric AMT configuration of Figure 1(b). The promised insensitivity to the dc-acceleration of this sequence, in practice, relies on the ability to accurately meet the condition of Eq. (2). In Figure 3(a), we probe the recombination of the main interferometer for the applied values of $\Delta \theta=10 \mathrm{mrad}$ and $20 \mathrm{mrad}$ by scanning the time shift $\Delta T_{\mathrm{s}}$ and recording the peak-peak contrast. 

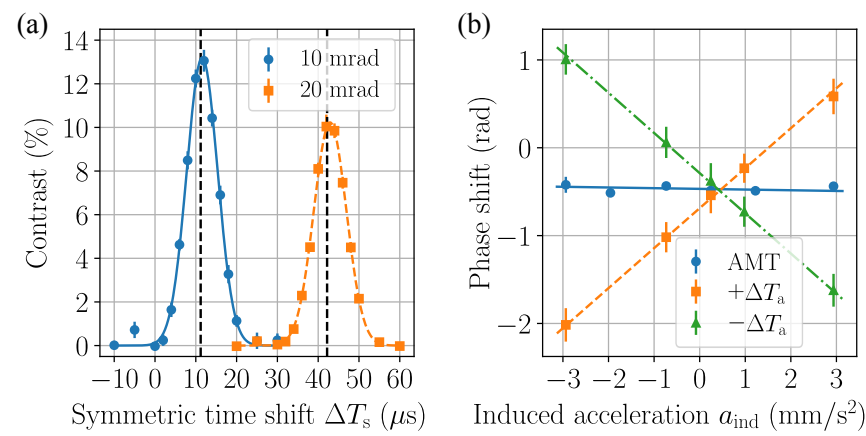

Figure 3. (a) Contrast of the main interferometer in the AMT configuration as a function of symmetric time shift $\Delta T_{\mathrm{s}}$, for two values of the angle $\Delta \theta$. The solid blue and dashed orange lines are empiric Gaussian fits to the data. The vertical dashed lines mark the expected center positions. (b) Phase shift as a function of induced acceleration (see text), in the AMT case with $\Delta \theta=20 \mathrm{mrad}$ and $\Delta T_{\mathrm{s}}=42.9 \mu \mathrm{s}$ (blue dots), and in the asymmetric case with $\Delta T_{\mathrm{a}}= \pm 40 \mu \mathrm{s}$ (orange squares and green triangles). Solid blue, dashed orange and dash-dotted green lines are linear fits to the data.

We find the peak centers at 11.6(1) $\mu$ s and 42.9(1) $\mu \mathrm{s}$, very close to their respective expectation of Eq. (2) with $\epsilon=\epsilon_{\text {corr }}$ at $11.2 \mu \mathrm{s}$ and $42.2 \mu \mathrm{s}$.

We choose the AMT arrangement with $\Delta \theta=20 \mathrm{mrad}$ and $\Delta T_{\mathrm{s}}=42.9 \mu \mathrm{s}$ to verify the insensitivity of the main interferometer to the linear dc-acceleration. We induce an additional acceleration along the $k_{\text {eff-direction }}$ via controlled tilt of the sensor in X-Z plane by a small angle $\beta$ such that $a_{\text {ind }}=g \cos \theta_{0} \sin \beta$, and measure the corresponding phase shift (blue dots in Fig. 3(b)). The fitted residual linear slope $d \Phi / d a_{\text {ind }}=0.4(8.5) \mathrm{rad} /\left(\mathrm{m} \cdot \mathrm{s}^{-2}\right)$ (blue line in Fig. 3(b)) is compatible with zero within the error bar. For comparison, we perform an identical measurement in the asymmetric configuration $[11,13]$ where both mirror pulses are advanced $\left(\Delta T_{\mathrm{a}}=40 \mu \mathrm{s}\right.$, orange squares) or delayed $\left(\Delta T_{\mathrm{a}}=-40 \mu \mathrm{s}\right.$, green triangles). For this configuration, we extract the respective dc-acceleration-sensitivity slopes of $446(8) \mathrm{rad} /\left(\mathrm{m} \cdot \mathrm{s}^{-2}\right)$ and $-448(5) \mathrm{rad} /\left(\mathrm{m} \cdot \mathrm{s}^{-2}\right)$ matching within $5 \%$ the expectation of $d \Phi / d a_{\text {ind }}=2 T \Delta T_{\mathrm{a}} k_{\text {eff. }}$. The ratio of the modulus of the slopes reflects a suppression of the accelerationinduced phase shift in a single measurement using the symmetric AMT configuration, as compared to the asymmetric one, by at least the factor of 50 .

We finally consider the impact of the AMT technique on the phase shift of the gyroscope sensor, which is given by (see Supplemental Material, section S4 [24]):

$$
\Delta \Phi=\frac{1}{2} \vec{k}_{\mathrm{eff}}(\vec{g} \times \vec{\Omega}) T^{3}\left(1-\frac{2 \epsilon}{3}\right)+\Delta \omega_{0} \frac{2 T \epsilon}{(1-\epsilon)} .
$$

The first term accounts for a correction to the gyroscope scale factor, as can be derived from the reduction of the physical (Sagnac) area of the interferometer, with $\vec{\Omega}$ being the rotation rate of the Earth. The second term (called hereafter clock shift) represents the sensitivity to the detuning $\left(\Delta \omega_{0}\right)$ of the relative Raman laser frequencies from the resonance condition of the Raman transition at the apogee point.

We measured the clock shift and confirmed the expected behavior of Eq. (6) (see Supplemental Material, section S4 [24]). By alternating measurements with $\pm k_{\text {eff }}$, we could demonstrate a rejection of this clock shift by at least two orders of magnitude, yielding a residual sensitivity for $\Delta \theta=20 \mathrm{mrad}$ compatible with zero and below $10 \mathrm{mrad} / \mathrm{kHz}$. The study of the correction to the gyroscope scale factor (first term of Eq. (6)) goes beyond the scope of this Letter.

To conclude, we have demonstrated the method of adjustable momentum transfer in multi-loop atom interferometers, that provides a controlled suppression of the spurious interferometric signals originating from the finite efficiency of atomic mirrors. The observed variation of the contrast of the spurious interferometers, quantitatively supported by our model, revealed a fractional imbalance in momentum transfer of $\epsilon \simeq 4 \times 10^{-5}$ to completely suppress the spurious signals, as given by the finite coherence of our atom source. In addition, we discovered a remarkable configuration $\left(\omega_{R} T \epsilon=\pi / 2\right)$, where the spurious interferometers are in anti-phase due to their different recoil sensitivity. The AMT method allowed us to demonstrate a double-loop gyroscope with a highly suppressed sensitivity to constant linear acceleration. This holds particular interest for applications where the fluctuations of the rotation rate of the ground need to be discriminated from the linear translations, as for example, in the field of rotational seismology [26].

Our results pave the way for the design of sensors with atomic sources of increased coherence or with more than two interferometric loops, where the problems associated with spurious paths are enhanced. More generally, our work shows the possibility of tuning the sensitivity of multi-loop atom interferometers to a unique, chosen, physical quantity, which enables to extend the scope of atom interferometry to new domains. This is crucial for multi-loop atom interferometers used as gravity gradiometers $[9,27]$ and gyroscopes $[11,13,28]$, or proposed for gravitational wave detection [29-32] or for measuring space-time curvature [33].

\section{ACKNOWLEDGMENTS}

We thank Peter Wolf and Albert Roura for stimulating discussions and Franck Pereira dos Santos for careful reading of the manuscript. We acknowledge the financial support from Ville de Paris (project HSENSMWGRAV), FIRST-TF (ANR-10-LABX-48-01), Centre National d'Etudes Saptiales (CNES), Sorbonne Universités (project SU-16-R-EMR-30, LORINVACC) and Agence Nationale pour la Recherche (project PIMAI, ANR-18-CE47-0002-01). L.A.S. was funded by Conseil Scientifique de l'Observatoire de Paris (PSL fellowship in astrophysics at Paris Observatory), M.A. and R. Gau- 
tier by the EDPIF doctoral school.

[1] G. F. Missiroli, G. Pozzi, and U. Valdre, "Electron interferometry and interference electron microscopy," Journal of Physics E: Scientific Instruments 14, 649-671 (1981).

[2] H. Rauch, S.A. Werner, and P.E.H. Rauch, Neutron Interferometry: Lessons in Experimental Quantum Oxford in Asia. Historical Reprints (Clarendon Press, 2000).

[3] Alexander D. Cronin, Jörg Schmiedmayer, and David E. Pritchard, "Optics and interferometry with atoms and molecules," Rev. Mod. Phys. 81, 1051-1129 (2009).

[4] Rafal E. Dunin-Borkowski, Martha R. McCartney, Richard B. Frankel, Dennis A. Bazylinski, Mihály Pósfai, and Peter R. Buseck, "Magnetic microstructure of magnetotactic bacteria by electron holography," Science 282, 1868-1870 (1998), https://science.sciencemag.org/content/282/5395/1868.fu

[5] Markus Arndt, Nadine Dörre, Sandra Eibenberger, Philipp Haslinger, Jonas Rodewald, Klaus Hornberger, Stefan Nimmrichter, and Marcel Mayor, "Matter-wave interferometry with composite quantum objects," (2015), arXiv:1501.07770 [quant-ph].

[6] Hartmut Abele and Helmut Leeb, "Gravitation and quantum interference experiments with neutrons," New Journal of Physics 14, 055010 (2012).

[7] Remi Geiger, Arnaud Landragin, Sébastien Merlet, and Franck Pereira Dos Santos, "High-accuracy inertial measurements with cold-atom sensors," arXiv e-prints , arXiv:2003.12516 (2020), arXiv:2003.12516 [physics.atom$\mathrm{ph}]$.

[8] John F. Clauser, "Ultra-high sensitivity accelerometers and gyroscopes using neutral atom matter-wave interferometry," Physica B+C 151, 262-272 (1988).

[9] J. M. McGuirk, G. T. Foster, J. B. Fixler, M. J. Snadden, and M. A. Kasevich, "Sensitive absolute-gravity gradiometry using atom interferometry," Physical Review A 65 (2002), 10.1103/PhysRevA.65.033608.

[10] H. Y. Carr and E. M. Purcell, "Effects of diffusion on free precession in nuclear magnetic resonance experiments," Phys. Rev. 94, 630-638 (1954).

[11] J. K. Stockton, K. Takase, and M. A. Kasevich, "Absolute Geodetic Rotation Measurement Using Atom Interferometry," Physical Review Letters 107 (2011), 10.1103/PhysRevLett.107.133001.

[12] B. Dubetsky and M. A. Kasevich, "Atom interferometer as a selective sensor of rotation or gravity," Phys. Rev. A 74, 023615 (2006).

[13] I. Dutta, D. Savoie, B. Fang, B. Venon, C. L. Garrido Alzar, R. Geiger, and A. Landragin, "Continuous cold-atom inertial sensor with $1 \mathrm{nrad} / \mathrm{sec}$ rotation stability," Physical Review Letters 116 (2016), 10.1103/PhysRevLett.116.183003.

[14] D. Savoie, M. Altorio, B. Fang, L. A. Sidorenkov, R. Geiger, and A. Landragin, "Interleaved atom interferometry for high-sensitivity inertial measurements," Sci Adv 4, eaau7948 (2018).

[15] Mark Kasevich and Steven Chu, "Atomic interferometry using stimulated raman transitions," Phys. Rev. Lett. 67, 181-184 (1991).
[16] Ch.J. Bordé, "Atomic interferometry with internal state labelling," Physics Letters A 140, 10-12 (1989).

[17] M. Altorio, L. A. Sidorenkov, R. Gautier, D. Savoie, A. Landragin, and R. Geiger, "Accurate trajectory alignechmaints, in cold-atom interferometers with separated laser beams," Phys. Rev. A 101, 033606 (2020).

[18] B. Dubetsky, "Asymmetric mach-zehnder atom interferometers," (2017), arXiv:1710.00020 [physics.atom-ph].

[19] Albert Roura, "Circumventing heisenberg's uncertainty principle in atom interferometry tests of the equivalence principle," Phys. Rev. Lett. 118, 160401 (2017).

[20] G. D'Amico, G. Rosi, S. Zhan, L. Cacciapuoti, M. Fattori, and G. M. Tino, "Canceling the gravity gradient phase shift in atom interferometry," Phys. Rev. Lett. 119, 253201 (2017).

d[f21] Chris Overstreet, Peter Asenbaum, Tim Kovachy, Remy Notermans, Jason M. Hogan, and Mark A. Kasevich, "Effective inertial frame in an atom interferometric test of the equivalence principle," Phys. Rev. Lett. 120, 183604 (2018).

[22] R. Caldani, K. X. Weng, S. Merlet, and F. Pereira Dos Santos, "Simultaneous accurate determination of both gravity and its vertical gradient," Phys. Rev. A 99, 033601 (2019).

[23] Ch. J. Bordé, Ch. Salomon, S. Avrillier, A. van Lerberghe, Ch. Bréant, D. Bassi, and G. Scoles, "Optical ramsey fringes with traveling waves," Phys. Rev. A 30, 1836-1848 (1984).

[24] See the Supplemental Material for the calculation of the phase shift of the spurious interferometers and their contrast, for additional data on the spurious interferometers, and for details of the phase shift of the main, double-loop interferometer

[25] The minus sign in front of the $\Delta \theta_{0 y}^{2}$ indicates that the bottom collimator is more tilted than the top one in the $y$ (horizontal) direction.

[26] Celine Hadziioannou, Peter Gaebler, Ulrich Schreiber, Joachim Wassermann, and Heiner Igel, "Examining ambient noise using colocated measurements of rotational and translational motion," Journal of Seismology 16, 787-796 (2012).

[27] I. Perrin, Y. Bidel, N. Zahzam, C. Blanchard, A. Bresson, and M. Cadoret, "Proof-of-principle demonstration of vertical-gravity-gradient measurement using a singleproof-mass double-loop atom interferometer," Phys. Rev. A 99, 013601 (2019).

[28] B. Canuel, F. Leduc, D. Holleville, A. Gauguet, J. Fils, A. Virdis, A. Clairon, N. Dimarcq, Ch. J. Bordé, A. Landragin, and P. Bouyer, "Six-axis inertial sensor using coldatom interferometry," Phys. Rev. Lett. 97, 010402 (2006).

[29] Jason M. Hogan, David M. S. Johnson, Susannah Dickerson, Tim Kovachy, Alex Sugarbaker, Sheng-wey Chiow, Peter W. Graham, Mark A. Kasevich, Babak Saif, Surjeet Rajendran, Philippe Bouyer, Bernard D. Seery, Lee Feinberg, and Ritva Keski-Kuha, "An atomic gravitational wave interferometric sensor in low earth orbit (agis-leo)," General Relativity and Gravitation 43, 1953-2009 (2011). [30] Peter W. Graham, Jason M. Hogan, Mark A. Kasevich, 
and Surjeet Rajendran, "Resonant mode for gravitational wave detectors based on atom interferometry," Phys. Rev. D 94, 104022 (2016).

[31] B. Canuel, A. Bertoldi, L. Amand, E. Pozzo di Borgo, T. Chantrait, C. Danquigny, M. Dovale Álvarez, B. Fang, A. Freise, R. Geiger, J. Gillot, S. Henry, J. Hinderer, D. Holleville, J. Junca, G. Lefèvre, M. Merzougui, N. Mielec, T. Monfret, S. Pelisson, M. Prevedelli, S. Reynaud, I. Riou, Y. Rogister, S. Rosat, E. Cormier, A. Landragin, W. Chaibi, S. Gaffet, and P. Bouyer, "Exploring gravity with the miga large scale atom interferometer," Scientific
Reports 8, 14064 (2018).

[32] C. Schubert, D. Schlippert, S. Abend, E. Giese, A. Roura, W. P. Schleich, W. Ertmer, and E. M. Rasel, "Scalable, symmetric atom interferometer for infrasound gravitational wave detection," (2019), arXiv:1909.01951 [quant$\mathrm{ph}$.

[33] Karl-Peter Marzlin and Jürgen Audretsch, "State independence in atom interferometry and insensitivity to acceleration and rotation," Phys. Rev. A 53, 312-318 (1996) 


\section{SUPPLEMENTAL MATERIAL FOR TAILORING MULTI-LOOP ATOM INTERFEROMETERS WITH ADJUSTABLE MOMENTUM TRANSFER}

\section{S1. PHASE SHIFTS OF THE SPURIOUS LOOPS}

Given a macroscopic separation of the two spurious interferometers of about $2.5 \mathrm{~mm}$ at the detection moment, they do not interfere between each other and may be considered separately. Below we derive the phase shift of the bottom spurious interferometer (Fig. S1) at the moment of detection. As the calculation for the top spurious loop is conceptually similar, we only provide the final result. In the following, we neglect the finite time length of the laser

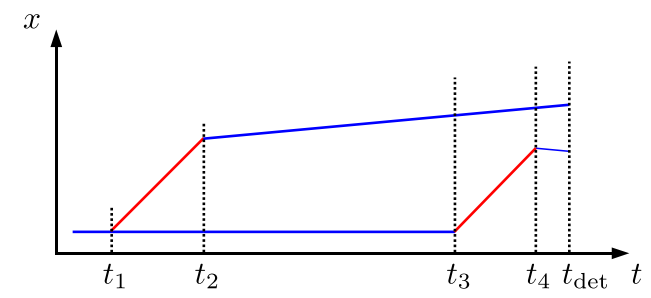

Figure S1. Sketch of the bottom spurious interferometer sequence until the detection moment. Vertical dashed lines indicate the four timings of the applied light pulses $\left\{t_{\mathrm{i}}\right\}$ and of the detection pulse. Red (blue) color labels $F=3(F=4)$ internal state of the atoms. For clarity, we show only the output port corresponding to the $F=4$ state.

pulses (all pulses have an area of $\pi / 2$ and time length of $10 \mu \mathrm{s}<<400 \mathrm{~ms}=T$ ) and consider them applied at the time moments $t_{1} . . t_{4}$ (see Fig. A1), while $t=0$ moment corresponds to the launch of the atomic cloud:

$$
\begin{aligned}
t_{1} & =114 \mathrm{~ms} \\
t_{2} & =t_{1}+T / 2-\Delta T \\
t_{3} & =t_{1}+3 T / 2+\Delta T+\Delta T_{3} \\
t_{4} & =t_{1}+2 T \\
t_{\operatorname{det}} & =t_{1}+2 T+\Delta t_{\text {det }},
\end{aligned}
$$

where $\Delta T=40 \mu \mathrm{s}$ is the initial time shift that separates in time the recombination moments of the main and spurious inerferometers (see main text and additional data section below), $\Delta T_{3}$ is the delay of the third pulse and $\Delta t_{\text {det }}=70 \mathrm{~ms}$ is the time interval past the last laser pulse until the detection moment $\left(t_{\mathrm{det}}\right)$.

The total interferometric phase shift may be represented as a sum of three parts [1]:

$$
\Delta \Phi=\Delta \Phi_{\text {las }}+\Delta \Phi_{\text {prop }}+\Delta \Phi_{\text {sep }}
$$

Here $\Delta \Phi_{\text {las }}$ is the laser phase which is imprinted onto the atomic wave-packet via interaction with Raman laser pulses; $\Delta \Phi_{\text {prop }}$ is the free propagation phase difference accumulated along the paths; $\Delta \Phi_{\text {sep }}$ is the phase shift arising from spatial separation of the two wave-packets at the moment of detection (interference), so-called separation phase. We take the convention for $\Delta \Phi$ being the phase shift of the upper branch minus the phase shift of the lower branch, and mark the related variables with $\mathrm{u}(\mathrm{l})$ subscripts.

Laser phase The laser phase reads as:

$$
\begin{aligned}
\Delta \Phi_{\text {las }} & =\left(\varphi_{1}-\varphi_{2}\right)_{\mathrm{u}}-\left(\varphi_{3}-\varphi_{4}\right)_{1} \\
\varphi_{1} & =\vec{k}_{\mathrm{eff}} \vec{r}_{\mathrm{u}}\left(t_{1}\right)-\int_{0}^{t_{1}} \omega_{\mathrm{eff}}(t) d t \\
\varphi_{2} & =(1-\epsilon) \vec{k}_{\mathrm{eff}} \vec{r}_{\mathrm{u}}\left(t_{2}\right)-\int_{0}^{t_{2}} \omega_{\mathrm{eff}}(t) d t \\
\varphi_{3} & =(1-\epsilon) \vec{k}_{\mathrm{eff}} \vec{r}_{1}\left(t_{3}\right)-\int_{0}^{t_{3}} \omega_{\mathrm{eff}}(t) d t \\
\varphi_{4} & =\vec{k}_{\mathrm{eff}} \vec{r}_{1}\left(t_{4}\right)-\int_{0}^{t_{4}} \omega_{\mathrm{eff}}(t) d t
\end{aligned}
$$


For completeness, we account here for the phase change due to the ramp of two-photon laser frequency to fulfill the resonance condition due to the Doppler shift: $\omega_{\text {eff }}(t)=\omega_{0}-\alpha\left(t-T-t_{1}\right)$, where $\omega_{0}=\omega_{h f}+\hbar k_{\text {eff }}^{2} / 2 m$ expresses the resonance condition for the atom at rest, namely at the apogee point of trajectory at $t=t_{1}+T$. The ramp rate $\alpha$ is given by the projection of the gravity acceleration on the Raman beams in $\Delta \theta=0$ configuration: $\alpha=k_{\text {eff }} g \sin \left(\theta_{0}\right)$. We also note that we orient the X-Z plane of our sensor (plane in which the area of the loops opens) towards the geographic West thus zeroing any possible contribution from the Earth rotation rate $\vec{\Omega}$.

We now consider an atom (wave-packet) with initial $(t=0)$ classical velocity $\vec{v}_{0}$ and position $\vec{r}_{0}$ and express the position of the wave-packet at the relevant time moments, along the upper and lower branch:

$$
\begin{aligned}
& \vec{r}_{\mathrm{u}}\left(t_{1}\right)=\vec{r}_{0}+\vec{v}_{0} t_{1}+\frac{1}{2} \vec{g} t_{1}^{2} \\
& \vec{r}_{\mathrm{u}}\left(t_{2}\right)=\vec{r}_{0}+\vec{v}_{0} t_{2}+\frac{1}{2} \vec{g} t_{2}^{2}+\frac{\hbar \vec{k}_{\mathrm{eff}}}{m}\left(t_{2}-t_{1}\right) \\
& \vec{r}_{1}\left(t_{3}\right)=\vec{r}_{0}+\vec{v}_{0} t_{3}+\frac{1}{2} \vec{g} t_{3}^{2} \\
& \vec{r}_{\mathrm{l}}\left(t_{4}\right)=\vec{r}_{0}+\vec{v}_{0} t_{4}+\frac{1}{2} \vec{g} t_{4}^{2}+\frac{\hbar \vec{k}_{\mathrm{eff}}}{m}(1-\epsilon)\left(t_{4}-t_{3}\right)
\end{aligned}
$$

We plug these formulae into the equations S3 and, with the use of explicit timings (Eqs. S1) and obtain the final result for the laser phase shift, where we neglect the terms of the orders of $O\left(\epsilon^{2}, \epsilon \Delta T / T, \epsilon \Delta T_{3} / T\right)$ :

$$
\begin{aligned}
& \Delta \Phi_{\mathrm{las}}=\Delta \Phi_{\mathrm{r}_{0}}+\Delta \Phi_{\mathrm{v}_{0}}+\Delta \Phi_{\mathrm{g}+\alpha}+\Delta \Phi_{\mathrm{rec}} \\
& \Delta \Phi_{\mathrm{r}}\left(r_{0}\right)=2 \vec{k}_{\mathrm{eff}} \vec{r}_{0} \epsilon \\
& \Delta \Phi_{\mathrm{v}}\left(v_{0}\right)=\vec{k}_{\mathrm{eff}} \vec{v}_{0}\left(2 \epsilon\left(T+t_{1}\right)-\Delta T_{3}\right) \\
& \Delta \Phi_{\mathrm{g}+\alpha}=\left(\vec{k}_{\mathrm{eff}} \vec{g}-\alpha\right)\left(\frac{3}{4} T^{2}-T \Delta T-\frac{3}{2} T \Delta T_{3}\right)+\vec{k}_{\mathrm{eff}} \vec{g}\left(\epsilon\left(\frac{5}{4} T^{2}+2 T t_{1}+t_{1}^{2}\right)-t_{1} \Delta T_{3}\right) \\
& \Delta \Phi_{\mathrm{rec}}=-\frac{\hbar k_{\mathrm{eff}}^{2}}{m} \Delta T_{3}
\end{aligned}
$$

The terms $\Delta \Phi_{\mathrm{r}}\left(r_{0}\right)$ and $\Delta \Phi_{\mathrm{v}}\left(v_{0}\right)$ depend on the initial position and velocity of the wave-packet. The last term $\Delta \Phi_{\mathrm{g}+\alpha}$ contains the dc-acceleration shift which is in the leading order compensated by the frequency ramp $\alpha$.

Free propagation phase The free propagation phase is given by the integrals of the Lagrangian along the two corresponding classical paths [2]:

$$
\begin{aligned}
& \Delta \Phi_{\text {prop }}=\int_{u} L(t) d t-\int_{1} L(t) d t \\
& L(t)=\frac{1}{2} m v(t)^{2}-m \vec{g} \vec{r}(t)
\end{aligned}
$$

Considering as before an atom (wave-packet) with initial classical velocity $\vec{v}_{0}$ and position $\vec{r}_{0}$, we perform straightforward integration until the moment of detection and obtain (neglecting same higher-order terms as in the calculation for the laser phase):

$$
\Delta \Phi_{\text {prop }}=\vec{k}_{\text {eff }} \vec{v}_{0}\left(\Delta T_{3}+2 \epsilon\left(T+\Delta t_{\text {det }}\right)\right)+\frac{\hbar k_{\text {eff }}^{2}}{2 m}\left(\Delta T_{3}+\epsilon T\right)
$$

Separation phase This contribution arises from the fact that one detects the interference between two wave-packets at a given location $\vec{r}$ in the detection region which has certain distances from the positions of the two classical trajectory points $\vec{r}_{\mathrm{u}}\left(t_{\mathrm{det}}\right)$ and $\vec{r}_{\mathrm{l}}\left(t_{\mathrm{det}}\right)$. The general expression for this phase shift reads [3]:

$$
\begin{aligned}
\Delta \Phi_{\mathrm{sep}} & =\frac{1}{\hbar}\left(\vec{p}_{\mathrm{u}}\left(\vec{r}-\vec{r}_{\mathrm{u}}\right)-\vec{p}_{\mathrm{l}}\left(\vec{r}-\vec{r}_{\mathrm{l}}\right)\right)=\frac{1}{\hbar}\left(-\vec{p}_{c} \overrightarrow{\Delta r}+\overrightarrow{\Delta p}\left(\vec{r}-\vec{r}_{c}\right)\right) \\
\vec{p}_{c} & =\frac{\vec{p}_{\mathrm{u}}+\vec{p}_{\mathrm{l}}}{2}, \overrightarrow{\Delta p}=\vec{p}_{\mathrm{u}}-\vec{p}_{\mathrm{l}} \\
\vec{r}_{c} & =\frac{\vec{r}_{\mathrm{u}}+\vec{r}_{\mathrm{l}}}{2}, \overrightarrow{\Delta r}=\vec{r}_{\mathrm{u}}-\vec{r}_{\mathrm{l}}
\end{aligned}
$$


The phase shift expression should be then integrated over the detection plane to obtain the full signal. The integration leaves unaffected the term proportional to the wave-packet separation $\overrightarrow{\Delta r}$, while the contribution of the second term depends on the difference of momenta $|\overrightarrow{\Delta p}|$ and the dimension of the detection region $d$. Assuming the mean position $\vec{r}_{c}$ at the center of detection region, we can define the critical condition when this phase contribution changes the sign and thus start to rapidly vanish due to the averaging: $(|\overrightarrow{\Delta p}| / \hbar) \cdot(d / 2)=\pi / 2$. In our case, $|\overrightarrow{\Delta p}| / \hbar=$ $2 \epsilon k_{\mathrm{eff}}$ and $d=30 \mathrm{~mm}$, which gives a critical value of $\epsilon_{\text {crit }}=3.8 \cdot 10^{-6}$. In the region of $\epsilon \sim \epsilon_{\text {crit }}$ this contribution might cause some varying phase shift bias. Understanding these variations requires further modeling that is outside the scope of the present work. This bias is, however, suppressed by at least an order of magnitude for the region $\epsilon \simeq 10 \epsilon_{\text {crit }}=0.4 \cdot 10^{-4}$ that covers about $80 \%$ of the probed $\epsilon$-span. We therefore neglect this contribution and obtain:

$$
\Delta \Phi_{\mathrm{sep}}=-\frac{m \vec{v}_{0}}{\hbar}\left(\vec{r}_{\mathrm{u}}\left(t_{4}\right)-\vec{r}_{1}\left(t_{4}\right)+2 \epsilon \frac{\hbar \vec{k}_{\mathrm{eff}}}{m} \Delta t_{\mathrm{det}}\right)=-\vec{k}_{\mathrm{eff}} \vec{v}_{0}\left(\Delta T_{3}+2 \epsilon\left(T+\Delta t_{\mathrm{det}}\right)\right)
$$

Note, that this expression is identical to the first term of $\Delta \Phi_{\text {prop }}$ (Eq. (S7)) but with an opposite sign, as one may expect for a case of Lagrangian being quadratic in position and momentum [4]. In particular, the dependence in the timing between the final beam-splitter pulse and the detection, $\Delta t_{\text {det }}$, drops out when summing the two contributions.

We now combine all the results obtained above and express the full phase shift of the bottom spurious interferometer:

$$
\Delta \Phi^{(\mathrm{B})}=\Delta \Phi_{\mathrm{r}}\left(r_{0}\right)+\Delta \Phi_{\mathrm{v}}\left(v_{0}\right)+\Delta \Phi_{\mathrm{g}+\alpha}+\frac{\hbar k_{\text {eff }}^{2}}{2 m}\left(T \epsilon-\Delta T_{3}\right) \equiv \Delta \Phi_{\mathrm{r}_{0}}+\Delta \Phi_{\mathrm{v}_{0}}+\Delta \Phi^{\prime}-\frac{\hbar k_{\text {eff }}^{2}}{2 m} T \epsilon
$$

with $\Delta \Phi^{\prime}=\Delta \Phi_{\mathrm{g}+\alpha}+\frac{\hbar k_{\text {eff }}^{2}}{2 m}\left(2 T \epsilon-\Delta T_{3}\right)$ being the mean phase shift independent of the initial atomic position and velocity. A fully identical calculation for the top spurious loop retrieves the same dephasing in all but recoil parts:

$$
\Delta \Phi^{(\mathrm{T})}=\Delta \Phi_{\mathrm{r}}\left(r_{0}\right)+\Delta \Phi_{\mathrm{v}}\left(v_{0}\right)+\Delta \Phi_{\mathrm{g}+\alpha}+\frac{\hbar k_{\mathrm{eff}}^{2}}{2 m}\left(3 T \epsilon-\Delta T_{3}\right) \equiv \Delta \Phi_{\mathrm{r}_{0}}+\Delta \Phi_{\mathrm{v}_{0}}+\Delta \Phi^{\prime}+\frac{\hbar k_{\mathrm{eff}}^{2}}{2 m} T \epsilon
$$

The total phase shifts of the two spurious loops are thus slightly different, such that $\Delta \Phi^{(\mathrm{T})}-\Delta \Phi^{(\mathrm{B})}=\frac{\hbar k_{\text {eff }}^{2}}{m} T \epsilon$. This difference arises from the recoil terms in the free propagation contribution and vanishes for $\epsilon \rightarrow 0$.

\section{S2. CONTRAST OF THE SPURIOUS LOOPS}

The employed fluorescence detection in our apparatus does not discriminate the signals coming from two spurious loops. The total peak-peak contrast is therefore given by an incoherent sum of the two spurious signals. Considering the wave-packet with initial classical velocity $\vec{v}_{0}$ and position $\vec{r}_{0}$ we write:

$$
C=\left[\frac{C^{(\mathrm{B})}}{2} \cos \Delta \Phi^{(\mathrm{B})}+\frac{C^{(\mathrm{T})}}{2} \cos \Delta \Phi^{(\mathrm{T})}\right]_{\mathrm{pp}},
$$

with phase shifts $\Delta \Phi^{(\mathrm{B})}$ and $\Delta \Phi^{(\mathrm{T})}$ defined in Equations S10, S11 and [...] $]_{\mathrm{pp}}$ denoting the peak-peak variation, and $C^{(\mathrm{B})}\left(C^{(\mathrm{T})}\right)$ being the contrasts of the bottom (top) spurious interferometer. We introduce the mean contrast $C_{0}=\frac{\left(C^{(\mathrm{B})}+C^{(\mathrm{T})}\right)}{2}$, the contrasts imbalance $\Delta C_{0}=C^{(\mathrm{T})}-C^{(\mathrm{B})}$, the mean dephasing $\overline{\Delta \Phi}=\frac{\Delta \Phi^{(\mathrm{T})}+\Delta \Phi^{(\mathrm{B})}}{2}$, and recall that $\Delta \Phi^{(\mathrm{T})}-\Delta \Phi^{(\mathrm{B})}=\frac{\hbar k_{\text {eff }}^{2}}{m} T \epsilon$. The Equation S12 becomes:

$$
C=C_{0}\left[\cos \overline{\Delta \Phi} \cos \left(\frac{\hbar k_{\mathrm{eff}}^{2}}{2 m} T \epsilon\right)-\frac{\Delta C_{0}}{C_{0}} \sin \overline{\Delta \Phi} \sin \left(\frac{\hbar k_{\mathrm{eff}}^{2}}{2 m} T \epsilon\right)\right]_{\mathrm{pp}}
$$

The observed contrasts of both spurious loops results from the averaging over the same initial velocity and position distributions in the atomic cloud. Assuming fully uncorrelated normal velocity $\left(\propto e^{-v_{0}^{2} / 2 \sigma_{v}^{2}}\right)$ and position $\left(\propto e^{-r_{0}^{2} / 2 \sigma_{r}^{2}}\right)$ distributions, we average the velocity- and position-dependent parts of the mean phase $\overline{\Delta \Phi}$ in Eq. S13 and obtain normalized full peak-peak contrast as:

$$
\begin{aligned}
& \frac{C\left(\epsilon, \Delta T_{3}\right)}{2 C_{0}}=\exp \left(-\frac{\left(2 k_{\text {eff }} \sigma_{r} \epsilon\right)^{2}}{2}\right) \times \exp \left(-\frac{\left(k_{\text {eff }} \sigma_{v}\left(2 \epsilon\left(T+t_{1}\right)-\Delta T_{3}\right)\right)^{2}}{2}\right) \times \\
& \times \frac{1}{2}\left[\cos \Delta \Phi^{\prime} \cos \left(\frac{\hbar k_{\text {eff }}^{2}}{2 m} T \epsilon\right)-\frac{\Delta C_{0}}{C_{0}} \sin \Delta \Phi^{\prime} \sin \left(\frac{\hbar k_{\text {eff }}^{2}}{2 m} T \epsilon\right)\right]_{\mathrm{pp}}
\end{aligned}
$$


In Figure S2 we show the fit of the data with the general-case model of Eqn. S14, where mean peak contrast $C_{0}$, contrast imbalance $\Delta C_{0}$ and standard deviation $\sigma_{r}$ are free parameters (solid red line). We extract the values of $\sigma_{r}=0.51(2) \mathrm{mm}, 2 C_{0}=0.934(18)$ and $\Delta C_{0}=0.03(3) \%$. The value of $2 C_{0}<1$ simply accounts for the actual overestimation of the maximum contrast resulting from data normalization to the maximum of the recorded contrasts. The fitted contrast imbalance $\Delta C_{0}=0.03(3) \%$ is well compatible with zero. Comparing this fit with the fit by simplified model used in the main text (dashed black line, for $\Delta C_{0}=0$ ) shows a small difference around the contrast local minimum at $\epsilon=0.76 \cdot 10^{-4}$, without any change for the rest of the probed $\epsilon$-values. Thus, all the arguments presented in the main text remain true for the case of the fit with exact function accounting for small contrast imbalance of the two spurious interferometers.

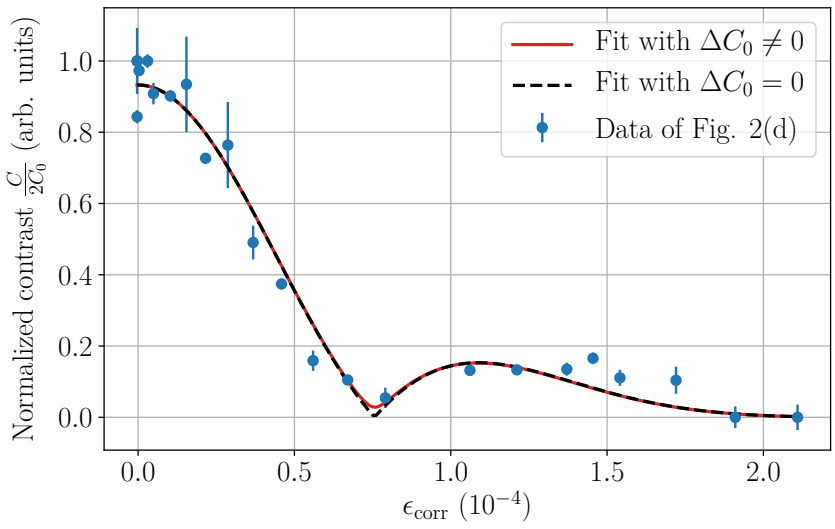

Figure S2. Normalized contrast of the spurious interferometers (data of Fig. 2(d), blue dots), fitted with exact model of Eqn. S14 (solid red line) and simplified model (Eqn. S14 with $\Delta C_{0}=0$, dashed black line), for comparison.

\section{S3. ADDITIONAL DATA ON SPURIOUS INTERFEROMETERS}

Time-domain width In Figure S3 we show the extracted the time-domain widths of the spurious interferometric peaks $\sigma_{t}$ for all data sets similar to those of the Figure 2(b). The data shows a rather large scatter for the probed range of $\Delta \theta$ which is likely to come from an hour-timescale experimental variations, and day-to-day drifts in case of different data sets. In overall, we cannot identify any clear systematic trend and the behavior seems consistent with the expected independence of $\Delta \theta$. We thus obtain a weighted mean value of $\bar{\sigma}_{t}=10.6(1.3) \mu \mathrm{s}$ (dashed black line in Fig. S3) that we use to empirically set the value of $\sigma_{v}=1 / k_{\text {eff }} \bar{\sigma}_{t}=1.8(2) v_{R}$, where $v_{R}$ is the single-photon atom recoil velocity. This value differs from the initial thermal width of 3.0(2) $v_{R}$, underlining the impact of the velocity-selection during the interrogation pulses.

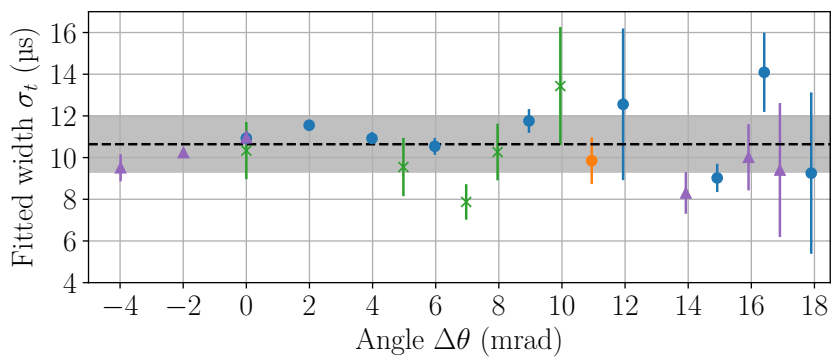

Figure S3. Fitted time-domain width of the spurious peaks for the probed values of $\Delta \theta$. Various symbols and accompanying colors indicate data sets taken on different days within two-week period. The dashed black line (gray-shaded area) are the weighted mean (standard deviation interval) of all shown data.

Time-separation of the spurious and main interferometers While studying controlled recombination of the spurious interferometers, it is important to verify that the main interferometer is sufficiently distant such that 
its wings do not affect the spurious signal. In Figure S4(a) we plot the expected peak recombination time moment for spurious (same as solid black line in Fig. 2(c) of the main text) and main interferometers. These functions are given by: $\Delta T_{3}=\left(T+t_{1}\right) \Delta \theta^{2}$ (spurious interferometer, solid blue line) and $\Delta T_{3}=-2 \Delta T+\frac{T}{2} \Delta \theta^{2}$ (main interferometer, dashed orange line), with $\Delta T=40 \mu \mathrm{s}$ being an initial time shift of the second and third pulses as explained in the main paper. The timing separation between two peaks, therefore, is minimal and equals $2 \Delta T$ for $\Delta \theta=0$ and increases with increasing $|\Delta \theta|$. In Figure $\mathrm{S} 4(\mathrm{~b})$ we demonstrate that the choice of $\Delta T=40 \mu$ s excludes any overlap between the two peaks for $\Delta \theta=0$.
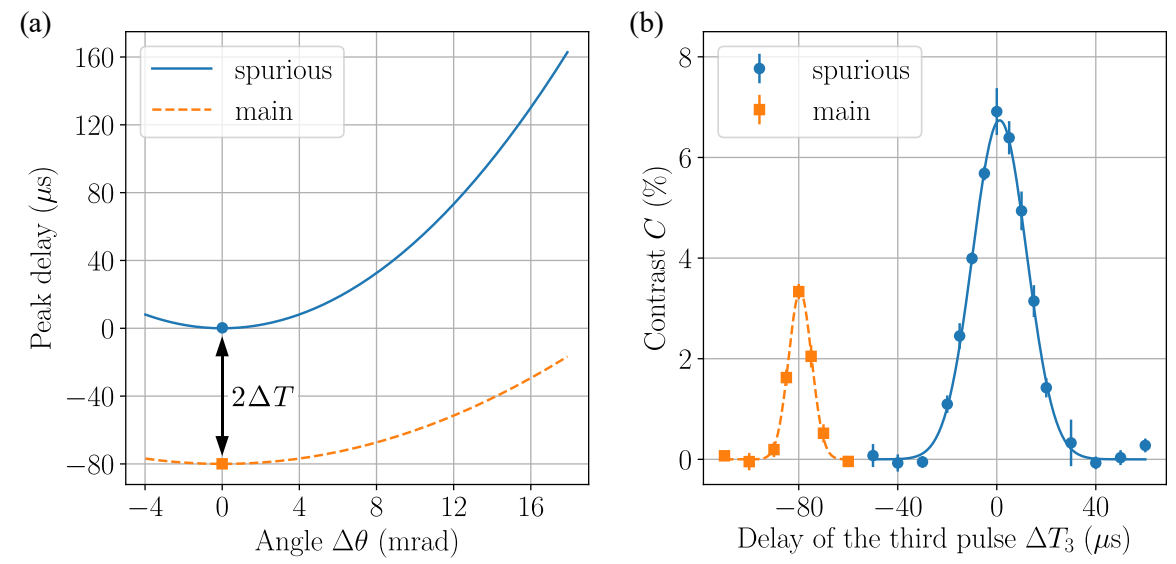

Figure S4. (a) Expected peak delay for spurious (solid blue line) and main (dashed orange line) interferometers as a function of $\Delta \theta$, for initial time separation $\Delta T=40 \mu \mathrm{s}$. The blue dot and orange square mark the expected peak positions for the data shown in the panel (b). (b) Peak-peak contrast of the spurious (blue dots) and main (orange squares) as a function of the third laser pulse delay $\Delta T_{3}$, for $\Delta \theta=0$, and Gaussian fits (solid blue and dashed orange lines) to the corresponding data.

\section{S4. PHASE SHIFT OF THE MAIN LOOP}

Sensitivity to rotation rate We derive the sensitivity to rotation rate of the main double-loop interferometer for the perfectly recombined symmetric configuration considered in the main paper using three different methods: the ABCD-matrix formalism [4], the full phase shift calculation approach (similar to the one of spurious intrferometers), and the geometric approach of Sagnac area calculation. All methods give the same result:

$$
\Delta \Phi_{\Omega}=\frac{1}{2} \vec{k}_{\mathrm{eff}}(\vec{g} \times \vec{\Omega}) T^{3}\left(1-\frac{2 \epsilon}{3}\right)
$$

Sensitivity to frequency An additional phase shift may arise in the AMT configuration if the effective laser frequency is detuned from the resonance condition at the apogee point of the fountain trajectory by a fixed amount $\Delta \omega_{0}$. This so-called clock shift can be estimated with by accounting for the frequency contribution to the imprinted laser phase (similarly to the above calculation for the spurious interferometers), or via sensitivity function [5] approach. In the limit of infinitely short laser pulses, we obtain:

$$
\Delta \Phi_{\text {clock }}=4 \Delta \omega_{0} \Delta T_{\mathrm{s}}=\Delta \omega_{0} \frac{2 T \epsilon}{(1-\epsilon)} \approx \Delta \omega_{0} T \Delta \theta^{2}
$$

To quantify the clock sensitivity, we record the induced phase shift from the controlled change of the two-photon detuning for a set of different angles. The phase shift $\Delta \Phi_{\mathrm{HS}}$ is evaluated as a half-sum (HS) of the measured values for alternating sign of $\vec{k}_{\text {eff }}$ and shows the expected linear dependence on frequency detuning $\Delta \omega_{0}$ (Fig. S5(a)). The fitted linear slopes $d \Delta \Phi_{\mathrm{HS}} / d\left(\Delta \omega_{0} / 2 \pi\right)$ scale quadratically with $\Delta \theta$ (blue dots in Fig. S5(b)), well matched with the expectation from Eq. S16 (solid black line). As the clock shift is independent on $\vec{k}_{\text {eff }}$, it should vanish (or be significantly suppressed) in the half-difference (HD) signal of $\pm k_{\text {eff }}$ method that leaves unaffected the inertial shifts. The orange squares in Figure S5(b) show the corresponding clock sensitivity given by $d \Delta \Phi_{\mathrm{HD}} / d\left(\Delta \omega_{0} / 2 \pi\right)$, boosted by a factor of 10 (including the error bars) for better visibility. We estimate a suppression factor ranging from about 10 (at $5 \mathrm{mrad}$ ) to better than 100 (at $20 \mathrm{mrad}$ ). 

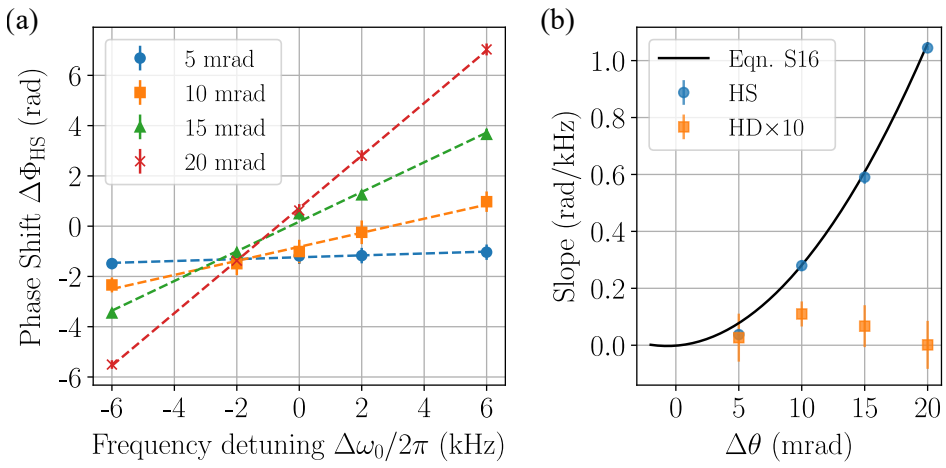

Figure S5. (a) Sensitivity of the main interferometer to the two-photon frequency in the AMT scheme, for different values of $\Delta \theta$, as extracted from the half-sum (HS) of the $\pm k_{\text {eff }}$ measurements. The solid blue, dashed orange, dash-dotted green and dotted red curves are linear fits to the corresponding data. (b) The fitted slopes $d \Delta \Phi / d\left(\Delta \omega_{0} / 2 \pi\right)$ of the half-sum signal of panel (a) and half-difference signal (HD), as a function of probed $\Delta \theta$. The solid black line indicates the expectation given by the Eqn. S16 for $\epsilon=\epsilon_{\text {calc }}$. The HD data and error bars are increased by a factor of 10 for visibility.

[1] Peter Wolf, Luc Blanchet, Christian J Bordé, Serge Reynaud, Christophe Salomon, and Claude Cohen-Tannoudji, "Does an atom interferometer test the gravitational redshift at the compton frequency?" Classical and Quantum Gravity 28, 145017 (2011).

[2] Pippa Storey and Claude Cohen-Tannoudji, "The feynman path integral approach to atomic interferometry. a tutorial." J. Phys. II France 4, 1999-2027 (1994).

[3] David Marvin Slaughter Johnson, Long baseline atom interferometry, Ph.D. thesis, Stanford University (2011).

[4] Ch. Antoine and Ch. J. Bordé, "Quantum theory of atomic clocks and gravito-inertial sensors: an update," Journal of Optics B: Quantum and Semiclassical Optics 5, S199-S207 (2003).

[5] P. Cheinet, B. Canuel, F. Pereira Dos Santos, A. Gauguet, F. Yver-Leduc, and A. Landragin, "Measurement of the sensitivity function in a time-domain atomic interferometer," IEEE Transactions on Instrumentation and Measurement 57, 1141-1148 (2008). 